\title{
Extending A Correction Method for Unsteady Transonic Aerodynamics to Variable Camber Continuous Trailing Edge Flap
}

\author{
Upender K. Kaul* and Nhan T. Nguyen** \\ NASA Ames Research Center, USA.
}

In this study, we have extended our correction method for transonic aerodynamics ${ }^{1}$ to baseline and variable camber continuous trailing edge (VCCTEF) geometries of super-critical airfoils at cruise conditions. This correction method modifies Theodorsen theory, which is based on incompressible potential flow over a flat plate. The method is partly based on CFD RANS simulations of oscillating NASA generic transport model (GTM) baseline cruise and VCCTEF airfoil geometries in pitch and shows promise for addressing transonic flutter problems of aeroelasticity. In the earlier study ${ }^{1}$, the Theodorsen functions were modulated with non-linear functions of Mach number, $M$, and reduced frequency, $k$, and thus the correction was demonstrated for the symmetrical NACA0012 airfoil. In the present study, the modulation functions are also allowed to account for the effects of camber and thickness of the airfoil. This is demonstrated by applying the correction method to various symmetric NACA00xx airfoil geometries (NACA001, NACA0002, NACA0012, NACA0015 and NACA0018), the NASA GTM super-critical baseline and VCCTEF airfoil geometries, e.g., the circular airfoil, VCCTEF222. First results indicate that the correction method accounts for a large variation of thickness of airfoils and camber. The present correction method will guide the development of a new state space model for the VCCTEF system and eventually a new transfer function that will be incorporated in a new aeroelastic framework leading to an appropriate transonic flutter model for use in the future aircraft systems in development under the NASA Advanced Air Transportation Technologies (AATT) project.

\section{Introduction}

Transonic flutter is a topic of high interest for aircraft design. Doublet lattice methods are frequently used in flutter analysis that can accurately predict low-speed flutter where the flow is entirely subsonic at low Mach number ${ }^{2}$. Transonic flutter, on the other hand, is a pacing item in transport aircraft design. Many methods for transonic flutter have been developed ranging from doublet-lattice methods with steady state transonic correction to high-fidelity CFD coupled to structural dynamic models of wing structures. ${ }^{3-5}$ While the transonic flutter prediction can be more accurate with high-fidelity CFD methods ${ }^{6,7}$ than with unsteady potential flow methods, the computational cost is high. In many applications that involve design optimization with flutter constraints, the computational cost associated with high-fidelity CFD presents a barrier. Therefore, computationally efficient methods for transonic flutter prediction continue to be of high interest to the aircraft design community. In our previous work ${ }^{1}$, we proposed a method for extending the classical Theodorsen's theory of unsteady aerodynamics for incompressible flow ${ }^{8}$ to transonic flow. The method proposes a modified Theodorsen's function to correct for changes in the amplitude and phase shift of the circulatory lift due to transonic flow. The method derives, partly, from a reduced set of steady state CFD simulations that are made a priori, as an input to the new method. Unsteady CFD simulations are

* Computational Aerosciences Branch, NASA Advanced Supercomputing (NAS) Division; Associate Fellow, AIAA

** Intelligent Systems Division; Associate Fellow, AIAA 
performed using OVERFLOW ${ }^{9-11}$ for selected NACA00xx airfoils, NACA0001, NACA0002, NACA0012, NACA0015 and NACA0018, and cruise GTM geometries, baseline and VCCTEF222, to extend the correction. Fig. 1 illustrates the VCCTEF deployed on the NASA generic transport model (GTM). The correction method $^{1}$ was also extended to truss-braced wing (TBW $)^{12}$ to include the thickness and camber effects of the airfoils. The proposed method demonstrates good agreement with the unsteady transonic CFD simulation results. Using this method, computationally efficient methods for transonic flutter prediction can be developed by incorporating appropriate transonic corrections to the Theodorsen's function using a strip-theory approach.

\section{Method and Formulation}

In our previous study ${ }^{1}$, we have shown that the proposed correction method is useful for NACA0012 airfoil with a blunt trailing edge. The correction method is based on modification of the Theodorsen model, which requires that the Kutta condition be enforced at the sharp trailing edge, that the wake is flat and that the wake vortices convect downstream at $V_{\infty}$. We have shown in the present paper that this model is useful for a variety of airfoils, such as NACA series airfoils (NACA0001, NACA0002, NACA0012, NACA0015 and NACA0018) with blunt trailing edge as well as baseline cruise and VCCTEF geometries. If the flow separates tangentially from the top and bottom edges of the trailing edge face, the Kutta condition is practically obeyed.

The zero-normal velocity condition on the airfoil is satisfied by source/sink singularities on the airfoil. The pitching motion of the airfoil determines the strength of the source/sink distribution. This is similar to the mixing layer flow over a flat plate subject to sinuous oscillations imposed at the trailing edge, where the zero normal velocity on the flat plate is enforced by a vortex sheet whose density changes with time ${ }^{13}$. The pressure difference caused by the associated potential gives rise to the "non-circulatory lift", which excludes the effect of the wake on the lift on the airfoil. This non-circulatory lift is a result of the so-called apparent mass effect due to inertia associated with the airfoil motion. It reflects the pressure forces required to accelerate the fluid near the airfoil. This lift dominates for the case when the normal velocity due to the pitching motion is a strong function of time, for example, for higher values of reduced frequency. The vortex singularity distribution in the wake gives rise to the lift which together with the quasi-steady lift forms the "circulatory lift". The quasi-steady lift is generated if the normal velocity due to the pitching motion is almost time-independent, i.e., $\partial v / \partial t \approx 0$, and the wake does not contribute to the lift. So, for lower values of reduced frequency, the circulatory lift becomes dominant.

For oscillatory airfoil, the singularity distributions on the airfoil and in the wake change with time, but satisfy the two constraints (zero normal velocity on the airfoil and Kutta condition) for all time. For flutter problems, the angle of attack is assumed to be small enough so that the flow separates approximately tangentially from the trailing edge.

Referring to our previous paper ${ }^{1}$, in which the derivation of the correction method has been laid out, we summarize the results of the method as follows.

The amplitude and phase shift of the oscillating circulatory lift are computed as

$$
\begin{aligned}
&\left|C_{l_{c i r c}}-\bar{C}_{l}\right|=\left|\Delta C_{l_{c i r c}}\right|=C_{l_{\alpha}} \alpha_{0} \sqrt{\left[C_{F} F(k)-C_{G} G(k) \frac{e_{c}}{c} 2 k\right]^{2}+\left[C_{G} G(k)+C_{F} F(k) \frac{e_{c}}{c} 2 k\right]^{2}} \\
& \measuredangle\left(\Delta C_{l_{c i r c}}\right)=\tan ^{-1} \frac{C_{G} G(k)+C_{F} F(k) \frac{e_{c}}{c} 2 k}{C_{F} F(k)-C_{G} G(k) \frac{e_{c}}{c} 2 k}
\end{aligned}
$$

The phase shift is also given by

$$
\measuredangle\left(\Delta C_{l_{\text {circ }}}\right)=2 k \tau_{\text {lag }}
$$

where $\tau_{\text {lag }}$ is the lag time between the oscillating circulatory lift and the quasi-steady state lift which is expressed as

$$
\Delta \bar{C}_{l}=C_{l_{\alpha}} \alpha_{0} \sin 2 k \tau
$$

When the quasi-steady state lift reaches its maximum amplitude at some normalized time $\tau=\tau_{0}$ after the flow establishes a steady state, the oscillating circulatory lift reaches its maximum ampltitude later at $\tau=\tau_{0}-\tau_{\text {lag }}$. Thus, $\tau_{\text {lag }}<0$ when the oscillating circulatory lift lags the quasi-steady state lift. 
These two nonlinear equations can be solved for $C_{F}$ and $C_{G}$ if the amplitude and phase shift of the circulatory lift are given. CFD simulations are performed to compute the amplitude and phase shift of the NACA0001, NACA0002, NACA0012, NACA0015, NACA0018, baseline cruise and VCCTEf222 airfoil geometries. The solutions are given by

$$
\begin{aligned}
C_{F} & =\frac{\left|\Delta C_{l_{c i r c}}\right|\left(\cos \measuredangle\left(\Delta C_{l_{c i r c}}\right)+\frac{e_{c}}{c} 2 k \sin \measuredangle\left(\Delta C_{l_{c i r c}}\right)\right)}{c_{l_{\alpha}} \Delta \alpha F(k)\left[1+\left(\frac{e_{c}}{c} 2 k\right)^{2}\right]} \\
C_{G} & =\frac{\left|\Delta C_{l_{c i r c}}\right|\left(\sin \measuredangle\left(\Delta C_{l_{c i r c}}\right)-\frac{e_{c}}{c} 2 k \cos \measuredangle\left(\Delta C_{l_{c i r c}}\right)\right)}{c_{l_{\alpha}} \Delta \alpha G(k)\left[1+\left(\frac{e_{c}}{c} 2 k\right)^{2}\right]}
\end{aligned}
$$

Since we are interested in flutter problems encountered in cruise, given a cruising Mach number, the Reynolds number is fixed accordingly. Therefore, in the present study, we do not seek an explicit Reynolds number dependence of the correction functions $C_{F}$ and $C_{G}$, as given by Eq. 5 and Eq. 6 above.

In this study, the modulation functions, $C_{F}$ and $C_{G}$ are allowed to account for the thickness and camber effects also.

\section{CFD Methodology}

The correction method ${ }^{1}$ extended in this study is based on a small set of steady state CFD simulations. The slope of the $\mathrm{Cl}-\alpha$ curve is required as an input to the correction method, which is provided by CFD. A number of unsteady CFD simulations were made for NACA0001, NACA0002, NACA0012, NACA0015, NACA0018, baseline cruise and VCCTEF222 geometries corresponding to cruise Mach number of 0.755. A new set of simulations corresponding to NACA0012 was also accomplished with the same pitching conditions as for other NACA00xx geometries, for comparison purposes. Pitching motion for NACA00xx geometries is considered about zero mean angle of attack, $\bar{\alpha}=0$, and amplitude of oscillation, $\alpha_{0}=0.5 \mathrm{deg}$. For the baseline and VCCTEF222 airfoils, $\bar{\alpha}$ is considered to correspond to cruise $C_{l}$ of 0.5 , and $\alpha_{0}=0.5$, as for the NACA00xx airfoils. Reynolds-Averaged Navier-Stokes (RANS) solver, OVERFLOW, with the Spalart-Allmaras (SA) turbulence model $^{14}$ is used for CFD simulations. For the steady state, central differencing of inviscid terms in the Navier-Stokes equations and ARC3D diagonalized Beam-Warming scalar pentadiagonal scheme ${ }^{15,16}$ are used. For the unsteady simulations, HLLC upwind scheme along with the symmetric successive overrelaxation (SSOR) algorithm with subiterations are used.

For an average grid resolution, each 2-D CFD simulation takes over 20 hours of wall clock time using 480 processors on NAS supercomputer at NASA Ames Research Center. In contrast, the present correction method represents a major theoretical advance, which extends Theodorsen theory to give us a closed form solution to the unsteady transonic problem. Only three CFD solutions are needed to produce the lift curve slope that is an input to our correction method.

\section{Grid and Time Stepsize Sensitivity}

In our previous studies ${ }^{1,17,18}$, various grid and time-stepsize resolutions were used to ascertain a fine enough grid and time stepsize for a grid independent and a fine unsteady solution. Accordingly, grid resolution for the NACA0001, NACA0002 and NACA0012 airfoil geometries was selected as 500x145x3. For the NACA0015 and NACA0018 airfoils, a finer grid resolution, 660x166x3, was used that gave a grid independent solution. A

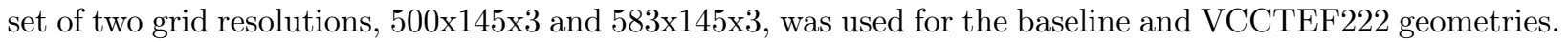
Grid independent solutions were obtained with these grid resolutions, i.e., both 500x145x3 and 583x145x3 grids yielded practically the same result.

\section{Results and Discussion}

All the unsteady transonic simulations were carried out at $\mathrm{M}=0.755$, with $\Delta \tau=0.001$. Fig. 2 (a,b,c) shows corrected model results for NACA0001 airfoil. Fig 1(a) shows a comparison of temporal evolution of circulatory lift amplitude corresponding to CFD and the quasi-steady case. To refresh, quasi-steady lift 
is that part of the circulatory lift for which the normal velocity due to pitching motion is almost timeindependent, i.e., $\partial v / \partial t \approx 0$, and to which there is no contribution from the wake. For $k=0.1$, considered here, circulatory lift is the dominant lift, which is captured well by CFD. Fig. 2(b) shows $C_{l}-\alpha$ hysteresis loops corresponding to Theodorsen theory, modified theory and CFD. An excellent agreement between CFD and the modified theory is shown. Clearly, there is a discrepancy between the Theodorsen theory and CFD. For the most part, this discrepancy is owing to the assumption in the Theodorsen theory of incompressible potential flow, since NACA0001 is a very thin airfoil. Fig. 2(c) shows temporal evolution of $C_{l}$. Again, excellent agreement is demonstrated between CFD and the modified theory. Discrepancy exists between CFD and Theodorsen theory, as expected.

Fig. 3(a,b,c) corresponds to the NACA0002 airfoil. Again, the modified theory predicts results vey well, as in the NACA0001 case. Fig. 4(a,b,c) shows results for NACA0012 airfoil. There is a difference between NACA0012 results ${ }^{1}$, where $\bar{\alpha}=0.016$ and $\alpha_{0}=2.51$, and the results shown in Fig 2(a,b,c); in the former case, the parameters do not correspond to flutter conditions. Rather, results ${ }^{1}$ were a demonstration of the robustness of the correction method, which worked even under more severe conditions than typical of flutter. Fig. 4(a), again, shows a comparison of circulatory lift amplitude between CFD and the quasi-steady case. Similar comparison is shown, as for NACA0002 airfoil. The $C_{l}-\alpha$ hysteresis loop is captured well by the modified theory, as compared to CFD. Again, an excellent agreement between the modified theory and CFD is shown for the $C_{l}$ time history in Fig. 4(c). For NACA0015, similar comparison is shown in Fig. 5(a,b,c). However, as the thickness increases further, while the comparison between the modified theory and CFD is good for NACA0018, as shown in Fig. 6(a,b,c), it is not excellent. It is expected, however, that if the amplitude of oscillation, $\alpha_{0}$ is decreased further, an excellent agreement will be recovered between CFD and the modified theory for NACA0018 also. It may be noted, however, typical thickness of cruise airfoil geometries is around $10-12$, which is way below the 18 thickness of NACA0018 airfoil.

For realistic airfoil transonic cruise geometries, such as those for NASA generic transport model (GTM), simulations were made corresponding to the baseline geometry and the VCCTEF222 airfoil geometry. A representative near body grid for VCCTEF222 is shown in Fig. 7. The modified theory performs equally well for these geometries also, as shown in the following discussion of results. Fig. 8(a,b,c) shows corresponding results for the baseline geometry, with a 500x145x3 grid resolution. An excellent agreement is shown between CFD and modified theory in terms of the $C_{l}-\alpha$ hysteresis loops and the time evolution of $C_{l}$ in Fig. 8(b) and Fig. 8(c), respectively. The baseline geometry was also simulated with the 583x145x3 grid resolution. The corresponding results are shown in Fig. 9(a,b,c). There is practically no difference in the predictions between the two grid resolutions. Same grid resolutions were used for the VCCTEF222 case also. Both yielded practically the same solution for VCCTEF222. In Fig. 10(a,b,c) are shown the corresponding results for the 500x145x3 grid for VCCTEF222. Again, an excellent agreement is shown in the $C_{l}-\alpha$ hysteresis loop and the temporal evolution of $C_{l}$ predictions between CFD and the modified theory.

Finally, effect of airfoil thickness, for airfoils considered, on the model coefficients, $C_{F}$ and $C_{G}$, is assessed. The airfoil thickness profiles are shown in Fig. 11, which shows all the geometries considered, i.e., NACA0001, NACA0002, NACA0012, NACA0015, NACA0018, baseline and VCCTEF222. The maximum thickness of baseline and VCCTEF222 is shown to be around 11 of chord, which is approximately close to that of NACA0012 airfoil. Clearly, NACA0015 and NACA0018 have thicker profiles than the baseline and VCCTEF222. Fig. 12(a,b) shows the variation of $C_{F}$ and $C_{G}$, respectively, in $k$, with thickness as a parameter. A clear dependence of $C_{F}$ and $C_{G}$ on thickness is shown, as the thickness of the airfoil increases. There is a monotonic variation in $C_{F}$ from NACA0001 to NACA0002 to baseline to NACA0012 to NACA0015, as the thickness increases. But, for NACA0018, this trend reverses. For $C_{G}$, the monotonic variation from NACA0001 to NACA0002 to baseline breaks trend at NACA0012 and starts to reverse at NACA0012. Similar behavior is shown through lift curves, $C_{l}-\alpha$, in Fig. 13(a). where the lift curve slope increases monotonically from NACA0001 to NACA0002 to baseline to NACA0012. But, a sudden change in the monotonic behavior occurs around NACA0015. This sudden reversal of results as the airfoil thickness increases will be the subject of a future study. Since the modified theory involving $C_{F}$ and $C_{G}$ is partly based on the steady state lift curve slope, this is also reflected in the $C_{F}$ variation in Fig. 12(a). Fig. 13(a) also shows that for the same thickness of baseline and VCCTEF222 airfoils, slope of the latter is smaller, which is exclusively due to the camber effect. Fig. 13(b) shows that the lift curve slope for all the airfoils considered is practically linear. 


\section{Concluding Remarks}

A correction method reported earlier ${ }^{1}$ has been extended to the NASA GTM super-critical airfoils. The correction method extends the classical Theodorsen's theory of unsteady aerodynamics of flat plate for incompressible flow to transonic flow over NASA GTM airfoils, namely, baseline and the circular airfoil, VCCTEF222. For cruise Mach number, $\mathrm{M}=0.755$, it is demonstrated that the modified theory works very well and is in excellent agreement with CFD. The modulation functions, $C_{F}$ and $C_{G}$, are shown to capture the effects of the airfoil thickness as well as the camber effect. The thickness effects are shown through different NACAxx configurations, and the camber effect in going from baseline to VCCTEF222 configuration. The modified theory is partly based on the results from the steady state CFD simulations. The present study demonstrates the viability of the correction method for the study of the transonic flutter problem of VCCTEF airfoils for a NASA GTM. The eventual goal is to devise a state space representation of the VCCTEF in transonic flutter and an associated transfer function that will readily be used in the study of aeroelasticity problems of interest.

\section{Acknowledgements}

This work was partially funded by the Advanced Air Transport Technology Project under the Advanced Air Vehicles Program of the NASA Aeronautics Research Mission Directorate (ARMD). The authors also would like to acknowledge Boeing Research and Technology and the University of Washington for their collaboration with NASA under NASA contract NNL11AA05B task order NNL12AD09T entitled "Development of Variable Camber Continuous Trailing Edge Flap System for B757 Configured with a More Flexible Wing."

OVERFLOW 2.2 was used in the present work for which the authors would like to thank Pieter Buning of NASA Langley Research Center.

\section{References}

[1] Kaul, U. K. and Nguyen, N. T., "A Correction Method for Unsteady Transonic Aerodynamics," AIAA2019-2037, AIAA SciTech 2019, San Diego, CA, January 2019

[2] Albano, E. and Rodden, W., "A Doublet-Lattice Method for Calculating Lift Distributions on Oscillating Surfaces in Subsonic Flows," AIAA Journal, Vol. 7, No. 2, February 1969, pp. 279-285.

[3] Bartels, R. E. and Stanford, B. K., "Aeroelastic Optimization with an Economical Transonic Flutter Constraint Using Naviertokes Aerodynamics," J. Aircraft, Vol. 55, No. 4, Julyugust 2018

[4] Mallik, W., Schetz, J. A. and Kapania, R. K., "Rapid Transonic Flutter Analysis for Aircraft Conceptual Design Applications," AIAA J., Vol. 56, No. 6, June 2018

[5] Max M. J. Opgenoord, Max M. J., Drela, M. and Willcox, K. E., "Physics-Based Low-Order Model for Transonic Flutter Prediction," AIAA J., Vol. 56, No. 4, April 2018

[6] Motta, V., Guardone, A. and Giuseppe, Q., "Influence of airfoil thickness on unsteady aerodynamic loads on pitching airfoils," J. Fluid Mech., Vol. 774, 2015, pp. 460-487

[7] Choi, J., Colonius, T. and Williams, D. R., "Surging and plunging oscillations of an airfoil at low Reynolds number," J. Fluid Mech., vol. 763, 2015, pp. 237-253. doi:10.1017/jfm.2014.674

[8] Theodorsen, T., "General Theory of Aerodynamic Instability and the mechanism of Flutter", NACA Report No. 496, 1949.

[9] Buning, P. G., "NASA OVERFLOW Overset Grid CFD Flow Solver," NASA Langley Research Center, Hampton, VA; 2016, https://overflow.larc.nasa.gov

[10] Buning, P. G., Gomez, R. J., and Scallion, W. I., 2004, "CFD Approaches for Simulation of Wing-Body Stage Separation," AIAA Paper No. 2004-4838. 
[11] Kandula, M., and Buning, P. G., "Implementation of LU-SGS Algorithm and Roe Upwinding Scheme in OVERFLOW Thin-Layer Navier-Stokes Code," AIAA Paper No. 94-2357, 1994.

[12] Nguyen, N., Fugate, J., Xiong, J. and Kaul, U., "Flutter Analysis of the Transonic Truss-BracedWing Aircraft Using Transonic Correction," AIAA Paper 2019-0217, AIAA SciTech Forum, January 2019, San Diego, California

[13] Kaul, U. K., "Do large structures control their own growth in a mixing layer ? An assessment," J. Fluid Mech., vol. 190, 1988, pp. 427-450

[14] Spalart, P. R., and Allmaras, S. R., "A One-Equation Turbulence Model for Aerodynamic Flows," AIAA 92-0439, AIAA 30th Aerospace Sciences Meeting and Exhibit, Reno, NV , January 1992.

[15] Beam, R., and Warming, R. F., 1976, "An Implicit Finite-Difference Algorithm for Hyperbolic Systems in Conservation Law Form," J. Comp. Phys., 22(1), pp. 8710.

[16] Pulliam, T. and Chaussee, D. R., 1976, "A Diagonal Form of an Implicit Approximate-Factorization Algorithm," J. Comp. Phys., vol. 39, no. 2,1981, pp. 347-363. ISSN 0021-9991

[17] Kaul, U. K. and Nguyen, N. T., "Drag Optimization Study of Variable Camber Continuous Trailing Edge Flap (VCCTEF) Using OVERFLOW," AIAA 2014-2444, 32nd Applied Aerodynamics Conference, Atlanta, GA, June 2014

[18] Kaul, U. K. and Nguyen, N. T., "Drag Characterization Study of Variable Camber Continuous Trailing Edge Flap," ASME J. Fluids Eng., Oct. 2018, Vol. 140, pp. 101108-1 


\section{Figures}

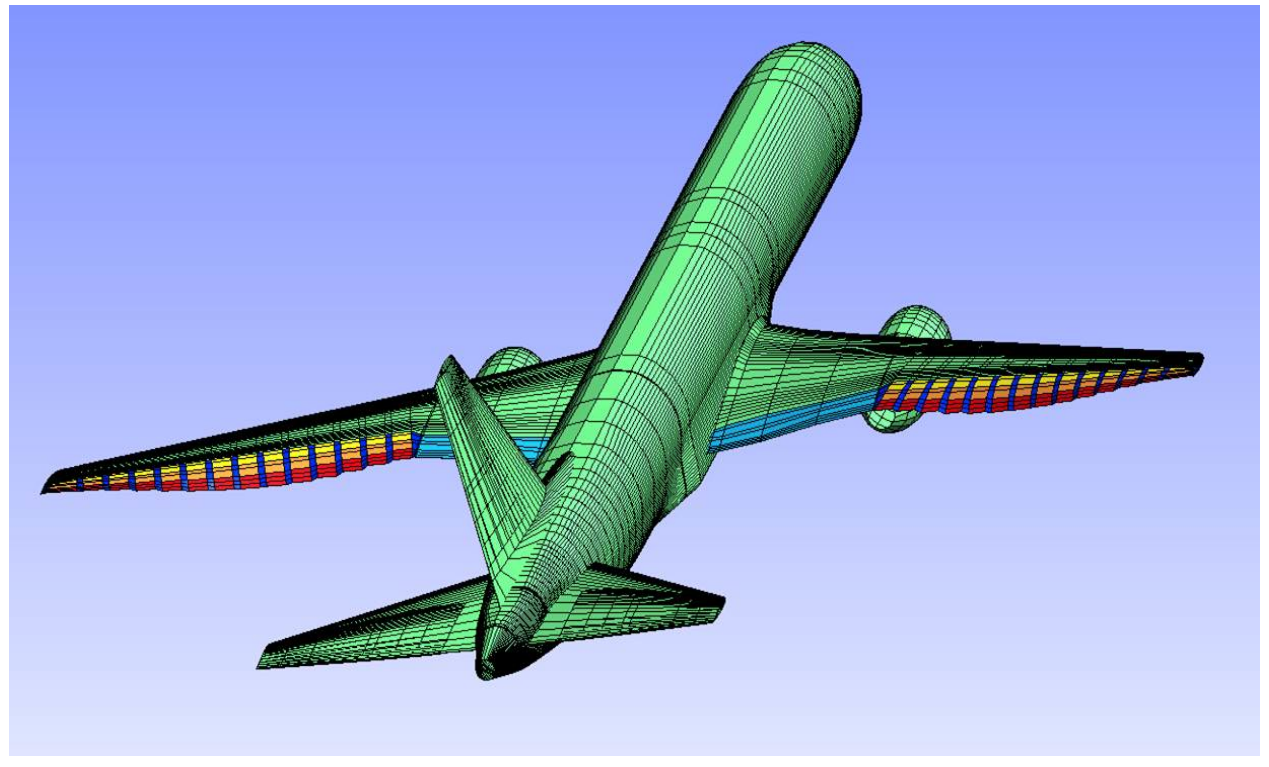

Figure 1: VCCTEF deployed on the generic transport model (GTM).

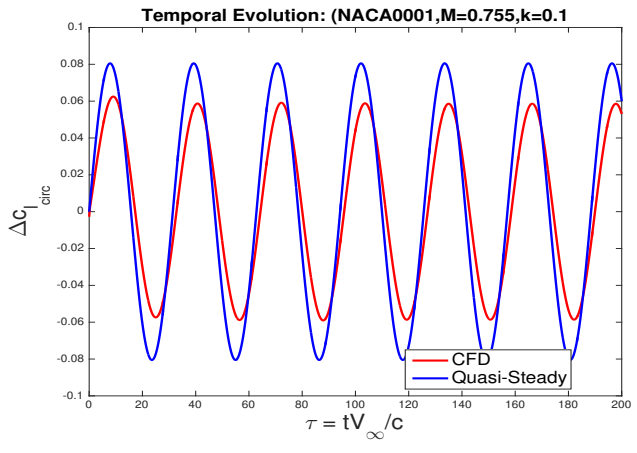

(a) Circulatory lift amplitude

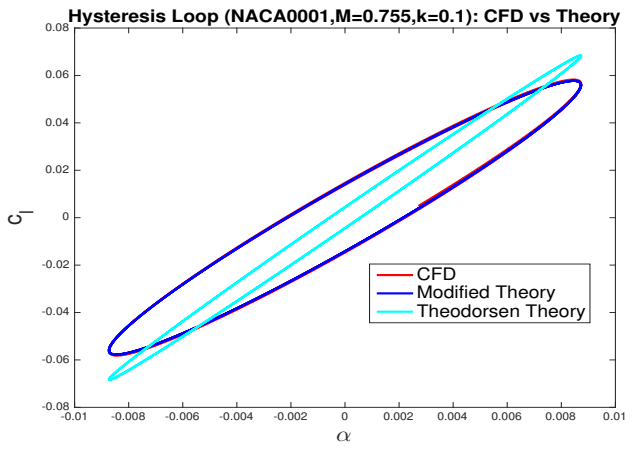

(b) Comparison of hysteresis loops

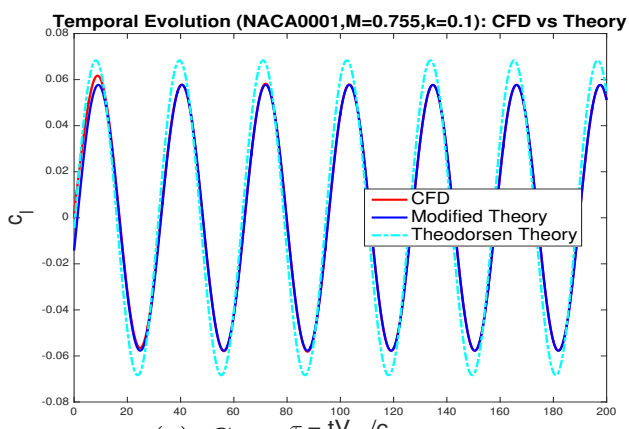

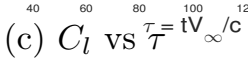

Figure 2: Correction method results for NACA0001 


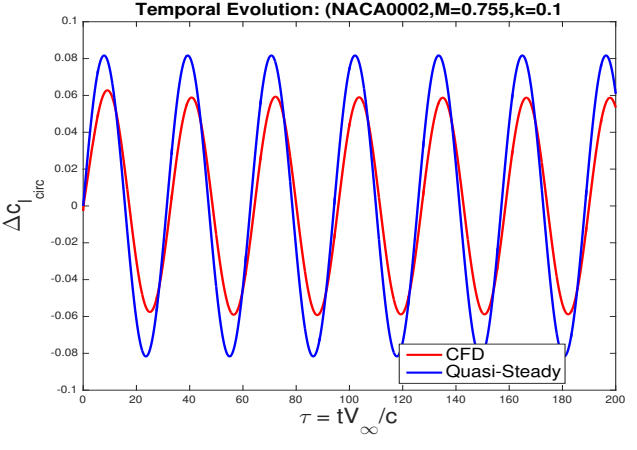

(a) Circulatory lift amplitude

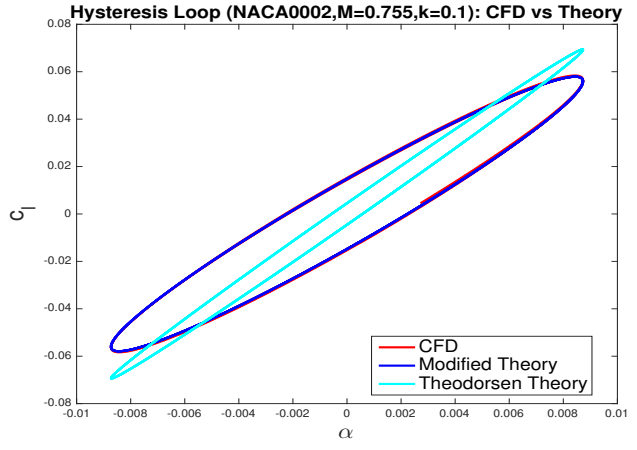

(b) Comparison of hysteresis loops

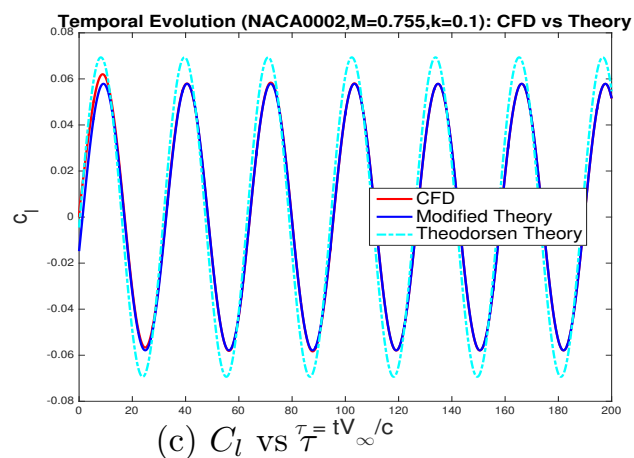

Figure 3: Correction method results for NACA0002 


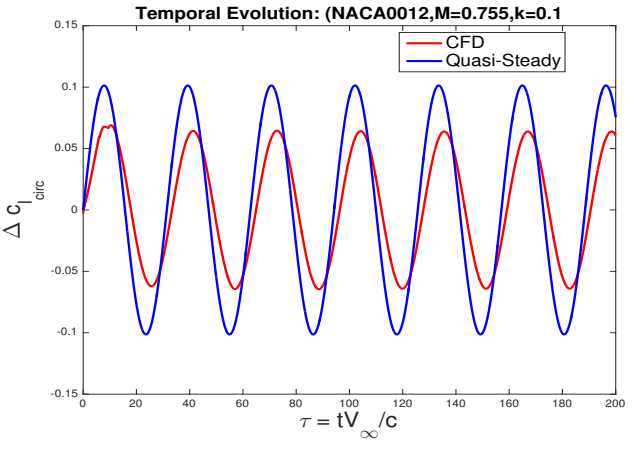

(a) Circulatory lift amplitude

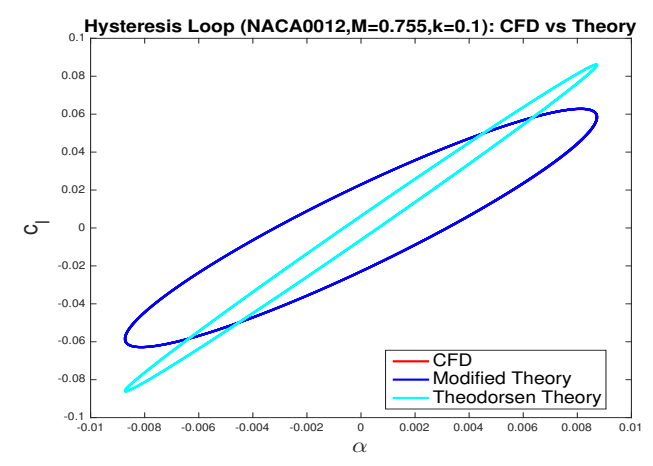

(b) Comparison of hysteresis loops

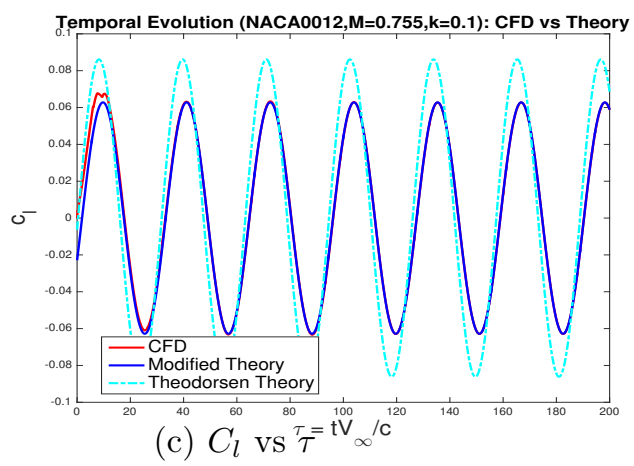

Figure 4: Correction method results for NACA0012 


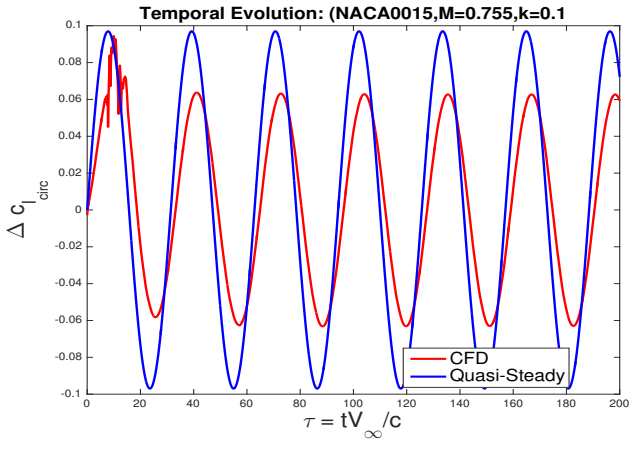

(a) Circulatory lift amplitude

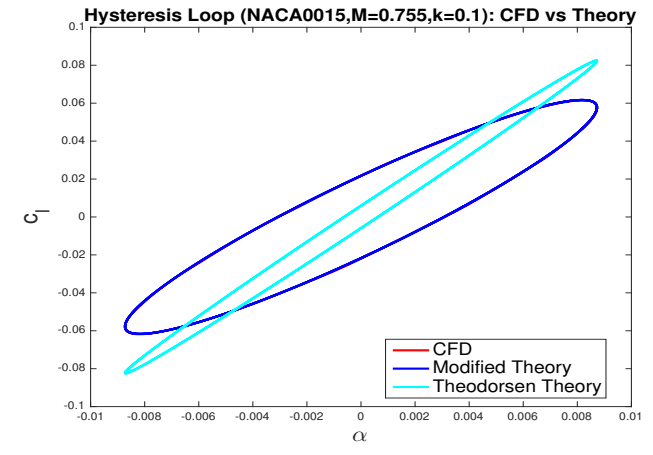

(b) Comparison of hysteresis loops

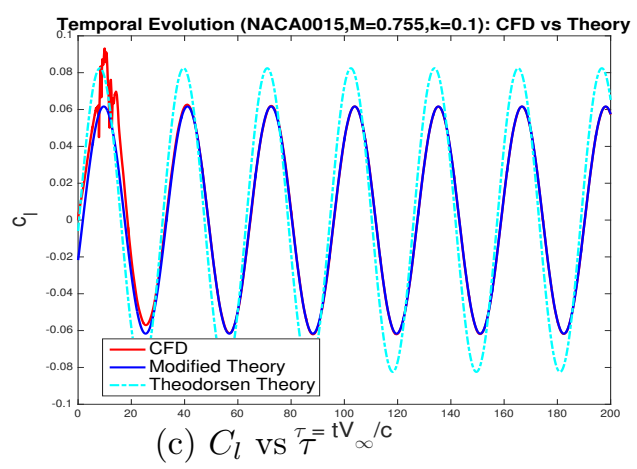

Figure 5: Correction method results for NACA0015 


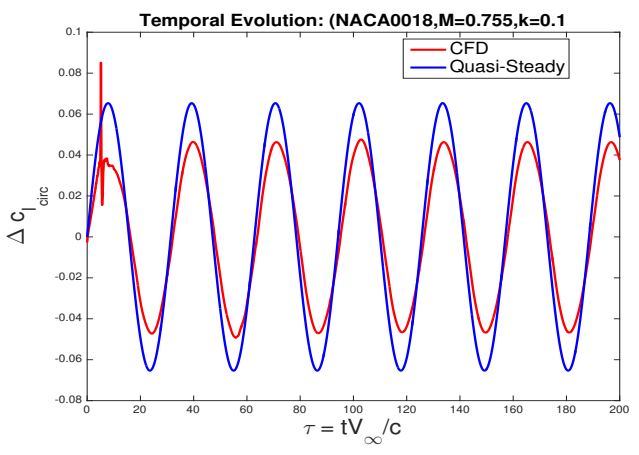

(a) Circulatory lift amplitude

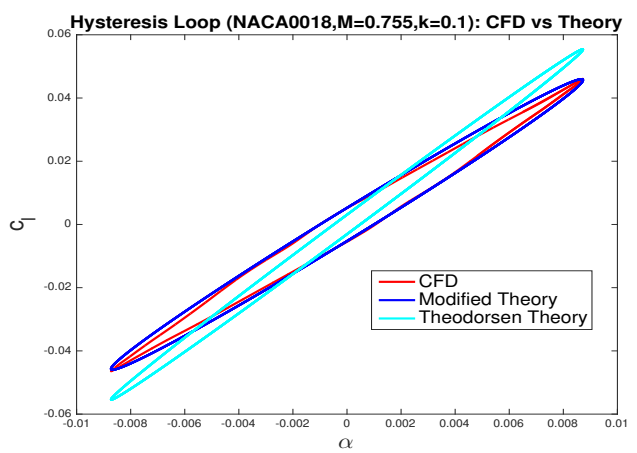

(b) Comparison of hysteresis loops

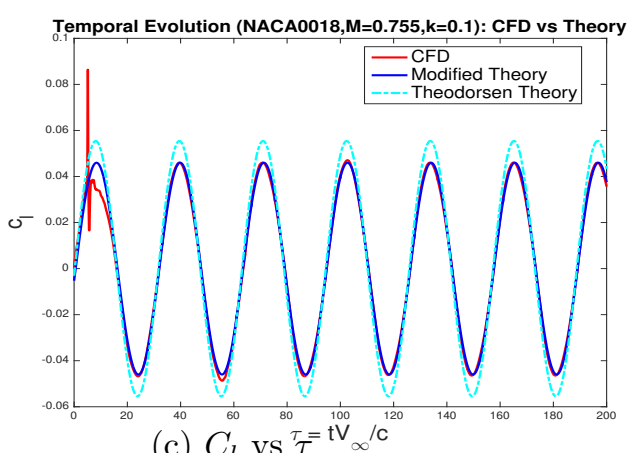

Figure 6: Correction method results for NACA0018

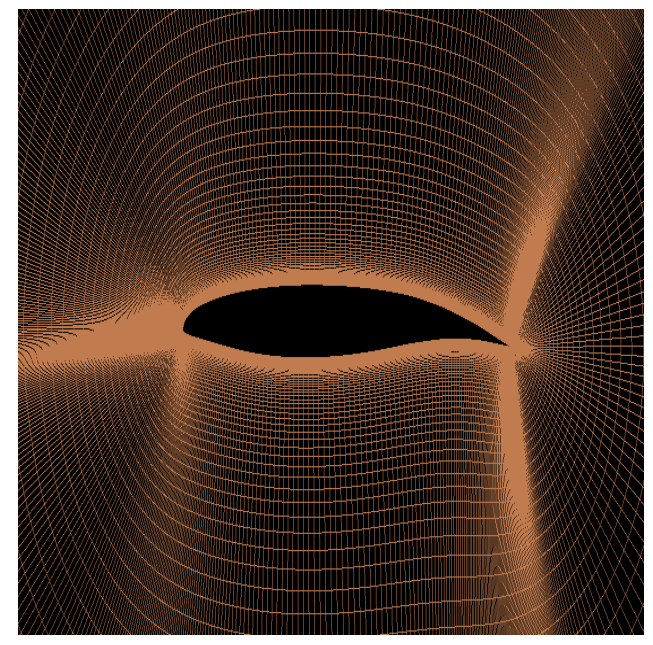

Figure 7: Representative grid for VCCTEF222: 583x145x3 grid resolution 


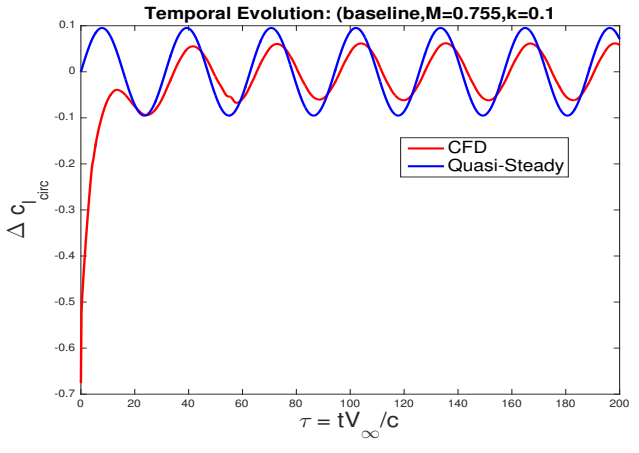

(a) Circulatory lift amplitude

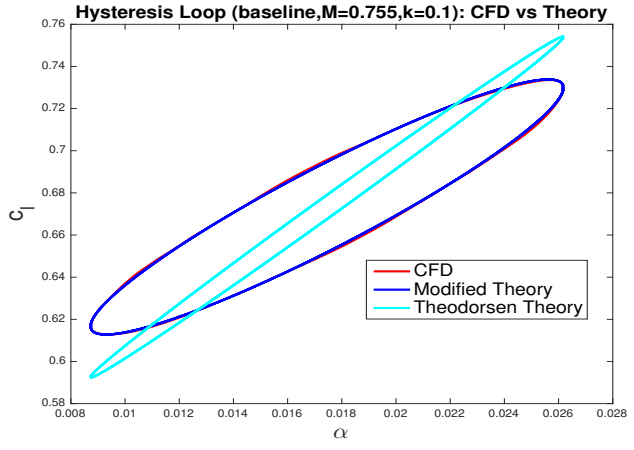

(b) Comparison of hysteresis loops

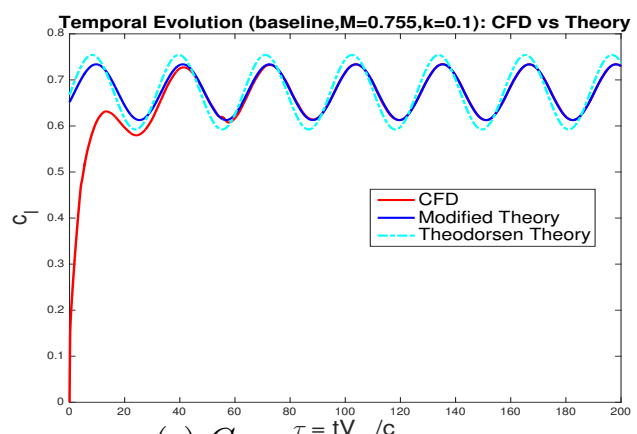

(c) $C_{l} \operatorname{vs}^{80}{ }^{\frac{80}{T}} \mathrm{tV}_{\infty}^{100}{ }_{\infty}^{120}$

Figure 8: Correction method results for baseline geometry with 500x145x3 grid resolution 


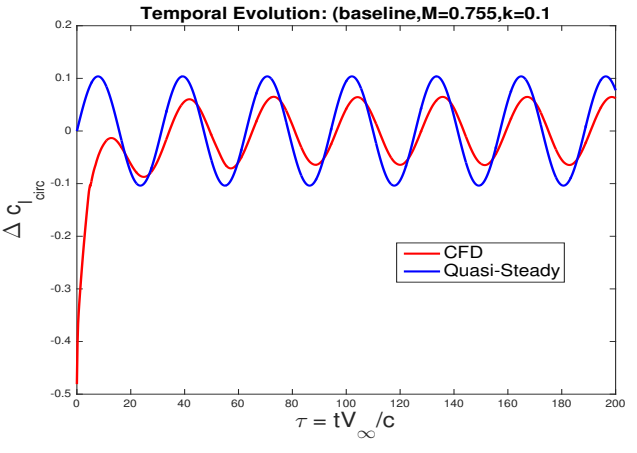

(a) Circulatory lift amplitude

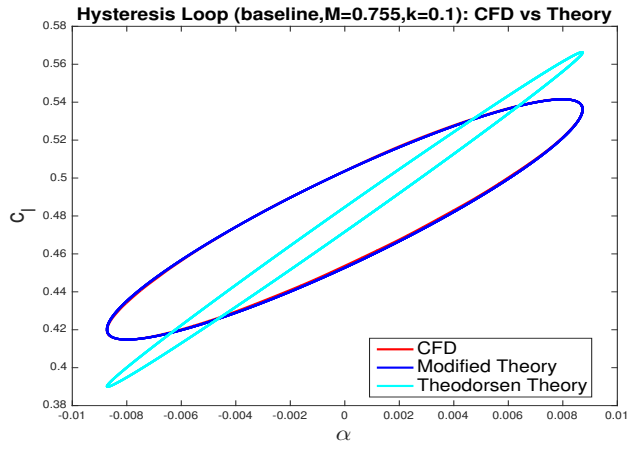

(b) Comparison of hysteresis loops

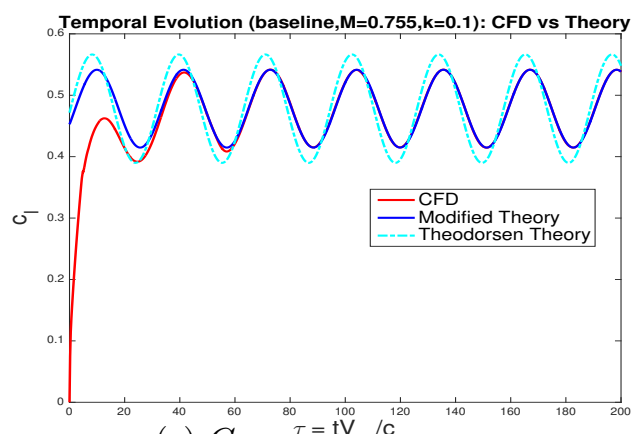

(c) $C_{l} \operatorname{vs}^{{ }^{80}}{ }^{T}=\mathrm{IV}_{\infty}^{100}{ }_{\infty}^{120}$

Figure 9: Correction method results for baseline geometry with 583x145x3 grid resolution 


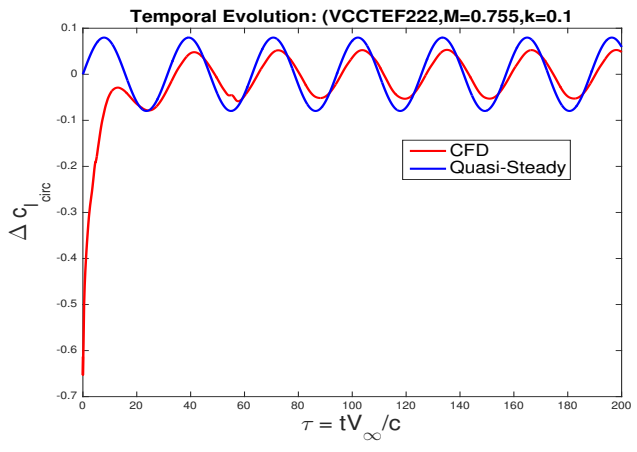

(a) Circulatory lift amplitude

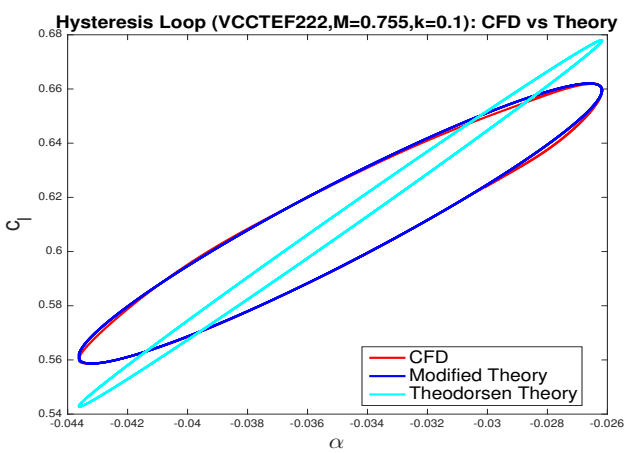

(b) Comparison of hysteresis loops

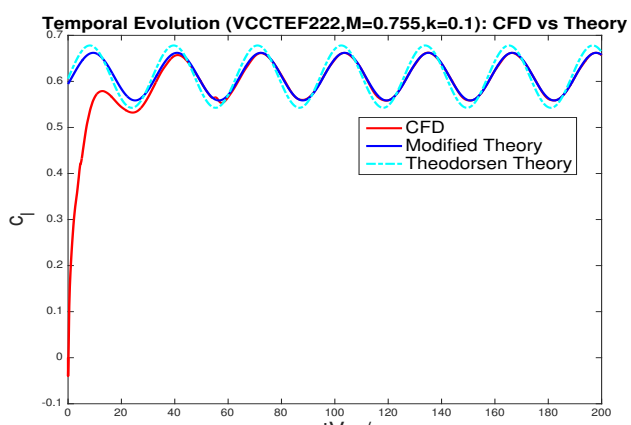

(c) $C_{l}{ }^{60} \operatorname{vs}^{80} \bar{\tau}^{100} \mathrm{tV}_{\infty}^{1 / c^{120}}$

Figure 10: Correction method results for VCCTEF222 with 500x145x3 grid resolution

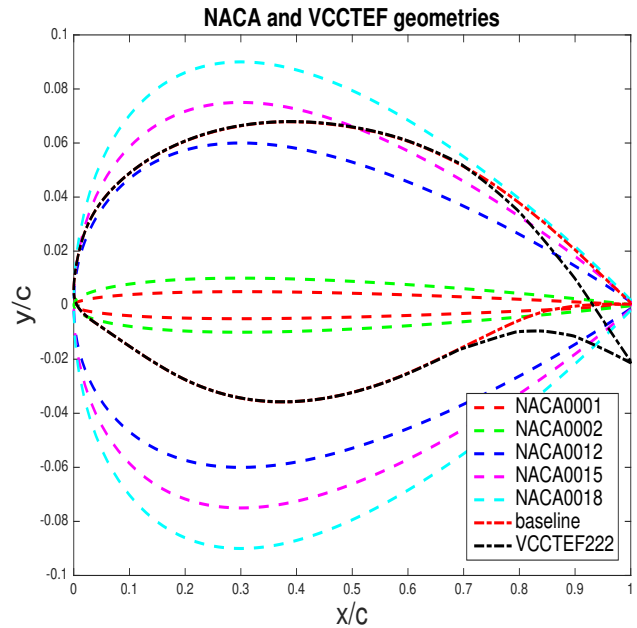

Figure 11: Thickness profiles for various geometries 


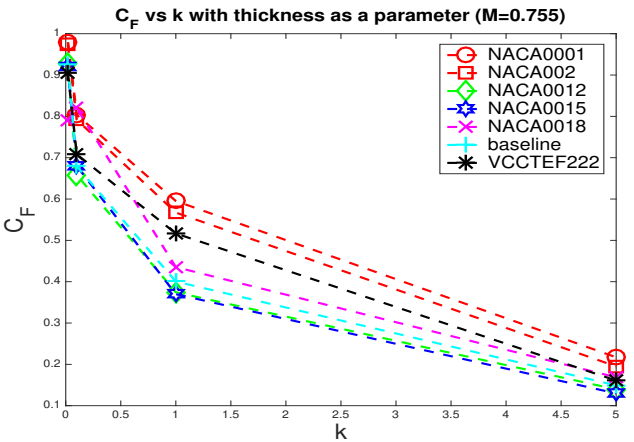

(a) Variation of correction function, $C_{F}$

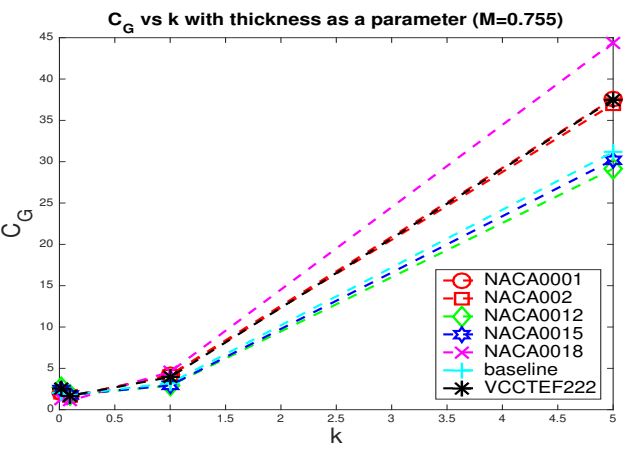

(b) Variation of correction function, $C_{G}$

Figure 12: Variation in $k$ of modified theory coefficients with thickness as a parameter

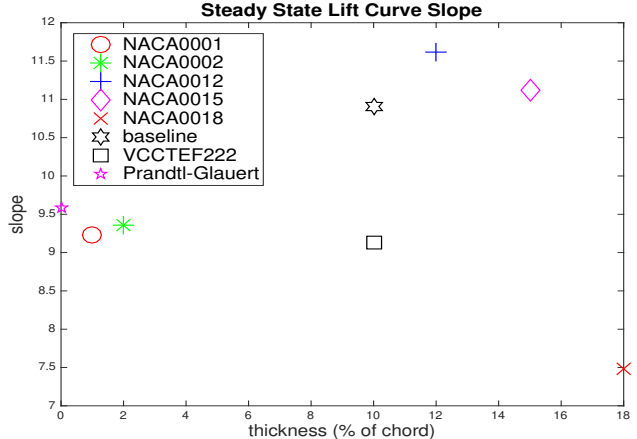

(a) Lift curve slope variation with thickness

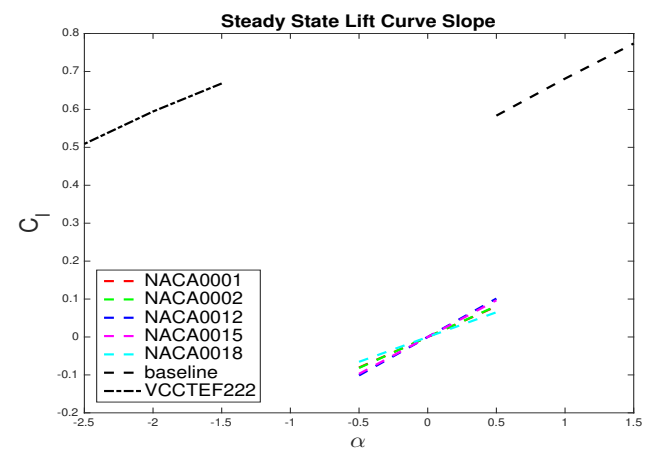

(b) Lift curve $C_{l}-\alpha$

Figure 13: Lift curve slope 